\title{
Relative intensity noise of Raman solitons: which one is more noisy?
}

\author{
Wei Liu, ${ }^{1}$ Gengji Zhou, ${ }^{1}$ JinKang Lim, ${ }^{2}$ Hung-Wen Chen, ${ }^{2}$ Franz X. Kärtner,,${ }^{1,23}$ and Guoqing Chang ${ }^{1,2}$ \\ ${ }^{1}$ Center for Free-Electron Laser Science, DESY, Notkestraße 85, D-22607 Hamburg, Germany \\ ${ }^{2}$ Research Laboratory of Electronics, Massachusetts Institute of Technology, 77 Mass Ave Cambridge MA 02139 \\ ${ }^{3}$ Physics Department, University of Hamburg, Luruper Chaussee 149, 22761 Hamburg, Germany \\ guoging.chang@desy.de
}

\begin{abstract}
We experimentally study the relative intensity noise of Raman solitons and find that earlier ejected Raman soliton exhibits lower noise. We also observe the bound soliton pair existing in a large range of excitation power.

(C)2014 Optical Society of America

OCIS codes: (190.4370) Nonlinear optics, fibers; (320.7110) Femtosecond phenomena.
\end{abstract}

Raman soliton self-frequency shift originates from the interplay between intra-pulse stimulated Raman scattering (SRS) and soliton formation in an optical fiber with anomalous dispersion. Raman soliton's center frequency red shifts continuously during the propagation and maintains its transform-limited hyperbolic secant profile. Capable of providing wavelength tunable femtosecond pulses at the desired wavelengths that cannot be directly obtained from mode-locked ultrafast lasers, Raman soliton sources have found important applications in spectroscopy and microscopy [1]. For enough pulse energy at the fiber's input, a higher-order soliton forms and then break into $N$ fundamental solitons mainly caused by higher-order dispersion. Differing in pulse duration and peak power, these $N$ fundamental solitons experience different amount of Raman-induced self-frequency shift, leading to multiple Raman solitons appearing at different center wavelength. Such a multicolor ultrafast laser source is of particular interest for multi-photon spectroscopy/microscopy applications or seeding into optical parametric amplifiers for high-field applications. Since the higher-order soliton fission and SRS involve strong nonlinearity, the relative intensity noise (RIN) of the input pulse may be amplified and distributed unequally among the multi- Raman solitons. In this paper, we carried out a detailed experimental study on the RIN of Raman solitons and found that earlier ejected Raman soliton has the lowest RIN- even significantly lower than the input RIN.

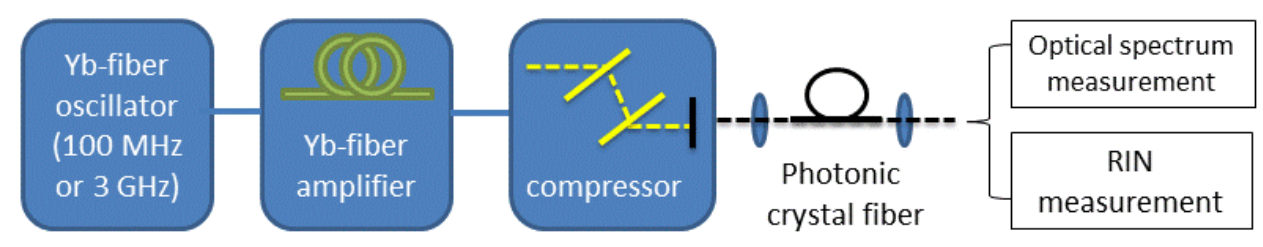

Fig. 1. Schematic setup to characterize the relative intensity noise (RIN) of Raman solitons. We carried out the experiment using 2 laser systems with different repetition rates: $100 \mathrm{MHz}$ and $3 \mathrm{GHz}$. The amplified pulses after an Yb-fiber amplifier are compressed by diffraction-grating compressor before coupled into a piece of photonic crystal fiber (PCF).

Figure 1 shows the schematic setup of Raman soliton sources based on two home-built Yb-fiber master-oscillatorpower-amplifier systems with different repetition-rates: $100 \mathrm{MHz}$ and $3 \mathrm{GHz}$. After a transmission-grating based compressor, both laser systems provide $\sim 110$ fs pulses with $>3 \mathrm{~nJ}$ pulse energy. To produce Raman solitons, we use a piece of photonic crystal fiber (PCF) with the zero-dispersion-wavelength at $945 \mathrm{~nm}$. The PCF has core diameter of $3.2 \mu \mathrm{m}$ and a nonlinear parameter of $23 \mathrm{~W}^{-1} / \mathrm{km}$. Figure 2 shows the experimental results for 3-m PCF pumped by the $100-\mathrm{MHz} \mathrm{Yb}$-fiber laser system. Figure 2(a) illustrates the spectra evolution at the PCF output as a function of the coupled average power. At 10-mW average power, the first Raman soliton appears and red shifts continuously with the increasing power, reaching $1.47 \mu \mathrm{m}$ at $134-\mathrm{mW}$ average power. The second Raman soliton emerges at 22$\mathrm{mW}$ average power and the third one at 46- $\mathrm{mW}$ average power. They form a bound soliton pair at 134-mW average power; that is, two fundamental solitons interact with each other forming a pair right after the soliton fission. Although SRS red shifts the center wavelength of the bound solitons, the time delay between them remains constant during propagation. Bound soliton has been studied recently using Ti:sapphire laser (peaking at $0.8 \mu \mathrm{m}$ ) pumping PCFs [2]. However it is the first experimental observation that such a bound Raman soliton pair exists in such a large power range - from $50 \mathrm{~mW}$ to $120 \mathrm{~mW}$. Beyond $120-\mathrm{mW}$ average power, the bound soliton pair breaks. At 134-mW average power, we filter out the $1^{\text {st }}(1.47 \mu \mathrm{m}), 2^{\text {nd }}(1.36 \mu \mathrm{m})$ and $3^{\text {rd }}(1.27 \mu \mathrm{m})$ Raman soliton and then measure their RIN separately. As Fig. 2(b) shows, the $1^{\text {st }}$ Raman soliton has the lowest RIN (green line); depending 
on the center wavelength, it can be nearly one order of magnitude less than the input pulse's RIN. The integrated RINs (black curves) corresponding to the $1^{\text {st }}, 2^{\text {nd }}$, and $3^{\text {rd }}$ Raman soliton are $0.075 \%, 0.23 \%$, and $1.22 \%$, respectively.
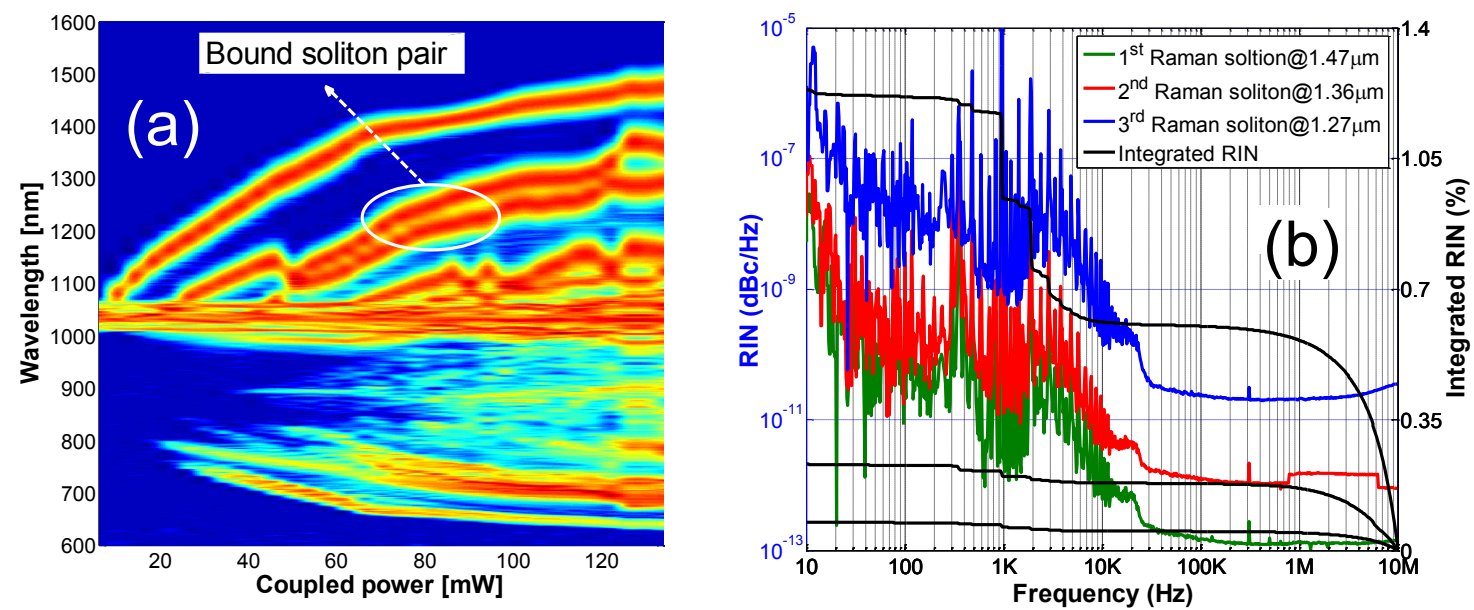

Fig. 2. Experimental results from the 100-MHz Yb-fiber laser system. (a) Measured output spectra versus coupled average power into 3-m PCF NL-3.2-945. Spectral intensity shown on a logarithm scale. (b) RIN measurement of the $1^{\text {st }}$ (green line), $2^{\text {nd }}$ (red line), and $3^{\text {rd }}$ (blue line) Raman solitons centered at $1.47 \mu \mathrm{m}, 1.36 \mu \mathrm{m}$, and $1.27 \mu \mathrm{m}$, respectively. These three Raman solitons are generated using 3-m PCF with $134 \mathrm{~mW}$ coupled average power. The three black curves represent the corresponding integrated RIN for the 3 Raman solitons.

We constructed a 3-GHz Raman soliton source and performed the similar measurements. Using multi-GHz laser system [3] to excite Raman solitons significantly increases the average power (by 30 fold to Watt level) of a Raman soliton source. Figure 2 (a) records the RIN for the $1^{\text {st }}$ and $2^{\text {nd }}$ Raman soliton, showing the $1^{\text {st }}$ Raman soliton is of low noise than the $2^{\text {nd }}$ one. We also measured the RIN of the $1^{\text {st }}$ Raman solitons centered at the same wavelength $(1.35 \mu \mathrm{m})$ resulted from a combination of different PCF length and coupled power. Figure 2(b) shows that using longer fiber with lower input power leads to the $1^{\text {st }}$ Raman soliton with lower RIN.
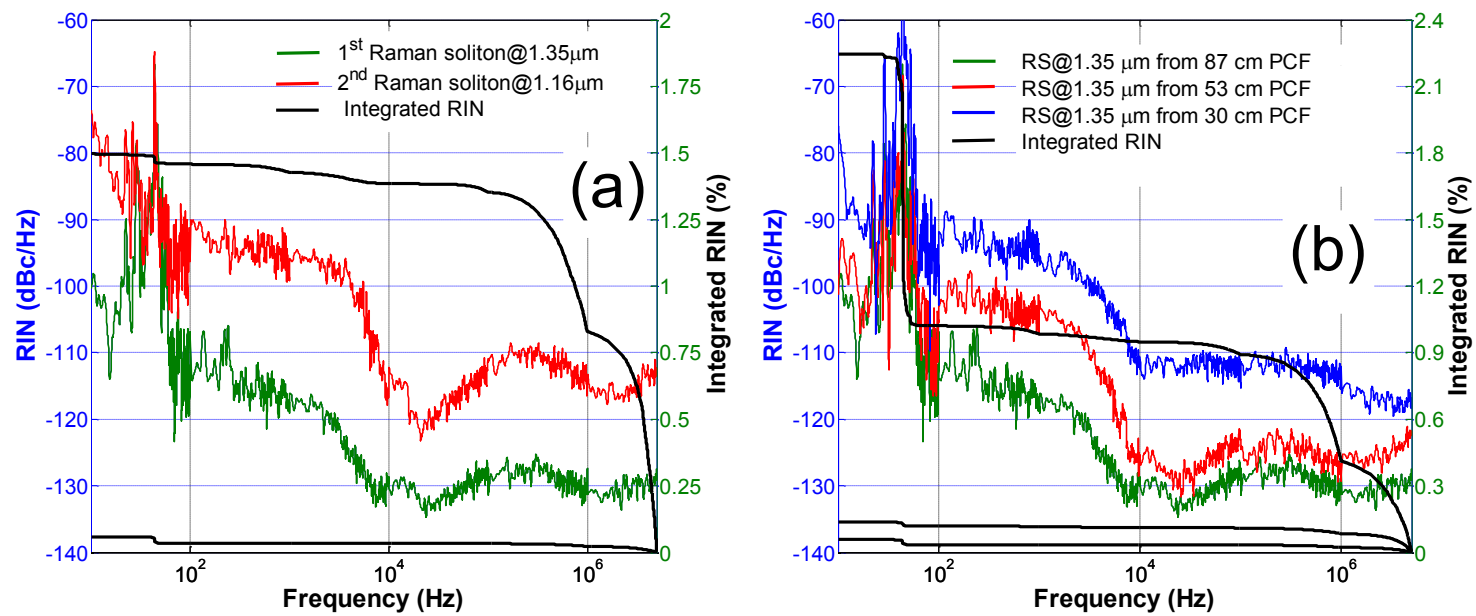

Fig. 3. Experimental results from the $3-\mathrm{GHz}$ Yb-fiber laser system. (a) RIN measurement of the $1^{\text {st }}$ (green line) and $2^{\text {nd }}$ (red line) Raman solitons centered at $1.35 \mu \mathrm{m}$ and $1.16 \mu \mathrm{m}$, respectively. These three Raman solitons are generated using 87-cm PCF with 1.3W coupled average power. Two black curves represent the corresponding integrated RIN for the two Raman solitons. (b) RIN measurement of the $1^{\text {st }}$ Raman solitons generated by different fiber lengths: $87 \mathrm{~cm}, 53 \mathrm{~cm}$, and $30 \mathrm{~cm}$. The coupled average powers are varied such that all these 3 Raman solitons peak at $1.35 \mu \mathrm{m}$. RS: Raman soliton.

\section{References}

[1] E. R. Andresen and H. Rigneault, Optical Fiber Technology 18, 379 (2012).

[2] A. Podlipensky et al., Opt. Express 15, 1653 (2017).

[3] H.-W. Chen et al., Opt. Lett. 37, 3522 (2012). 